\title{
Biomechanical analysis of the right elevated glenohumeral joint in violinists during legato-playing
}

\author{
Anne-Sophie Saffert ${ }^{\mathrm{a}, *}$, Maximilian Melzner ${ }^{\mathrm{a}}$ and Sebastian Dendorfer ${ }^{\mathrm{a}, \mathrm{b}}$ \\ ${ }^{a}$ Laboratory for Biomechanics, Ostbayerische Technische Hochschule (OTH) Regensburg, Regensburg, \\ Germany \\ ${ }^{\mathrm{b}}$ Regensburg Center of Biomedical Engineering, OTH and University Regensburg, Regensburg, Germany
}

Received 31 May 2021

Accepted 21 September 2021

\begin{abstract}
.
BACKGROUND: Many statistics reveal that violin players suffer most often from musculoskeletal disorders compared to musicians of other instrument groups. A common phenomenon, especially observed in violin beginners, is the tendency to elevate the right shoulder during playing the violin. This can probably lead to serious disorders in long-term practice with repetitive movements.

OBJECTIVE: For this reason, this study investigated the relationship between the right shoulder elevation and the force in the right glenohumeral joint during violin playing. It was hypothesized that the forces in the right glenohumeral joint are higher during playing with the right shoulder raised compared to playing in normal posture.

METHODS: Motion capture data from four experienced violinists was recorded and processed by means of musculoskeletal simulation to get the force and elevation angle while playing with raised shoulder and in normal position.

RESULTS: The results indicate that the absolute values of the resulting force, as well as the forces in the mediolateral, inferosuperior, and anteroposterior directions, are higher in playing the violin with the shoulder raised than in a normal posture. CONCLUSIONS: Elevating the right shoulder while playing the violin may pose a potential problem.
\end{abstract}

Keywords: Biomechanics, violin, shoulder elevation, shoulder joint force, musculoskeletal disease

\section{Introduction}

Performing Arts Medicine describes the science of health problems of artists. The term Musicians Medicine represents a subgroup of Performing Arts Medicine, which only considers instrumentalists. This subgroup has become more and more important over the years due to the growing music industry [1]. An essential aspect of Musicians Medicine is musculoskeletal disorders, which concern about $70 \%$ of all musicians [2]. Among them, the group of 26 to 35 years old is the most affected one [3]. Regarding the instrument groups, Rensing et al. [2] report that musculoskeletal disorders are most frequently

\footnotetext{
${ }^{*}$ Corresponding author: Anne-Sophie Saffert, Laboratory for Biomechanics, Ostbayerische Technische Hochschule (OTH) Regensburg, Galgenbergstraße 30, 93053 Regensburg, Germany. Tel.: +49 941943 5171; E-mail: as.saffert@ web.de.
} 
observed in string players with a percentage of $65 \%$ to $88 \%$. Especially among violinists such a disease is most common in the shoulder girdle [3,4]. Even rehabilitation procedures do not lead to a complete recovery in many cases [5]. In general, even young musicians suffer from playing-related musculoskeletal diseases [6,7]. In the worst case, a musculoskeletal disorder in instrumentalists can lead to a premature end of their professional musician career. About $12 \%$ of all classical professional musicians have to cancel their career due to these disorders as studies of Kaufman-Cohen et al. [8] show.

The string instruments include violin, viola, cello, and double bass. These instruments are similar in construction and playing, especially the posture and movement of the bow is approximately the same. The bow is normally controlled by the right arm, whereas the left arm is used for playing the keys on the strings. While playing, a straight posture should be adopted in standing as well as in sitting. Each string player has a slightly different appropriate posture. In general, concerning the shoulder girdle, it is important to ensure that the shoulders are relaxed and therefore not elevated (movement of the shoulder to cranial) or protracted (movement of the shoulder to anterior) [9]. Playing the violin with elevated shoulders can therefore be stated as a suboptimal posture. In the normal playing posture, the muscles trapezius, levator scapulae, and rhomboidei are primarily required [10]. The upstroke is defined as the upward movement of the bow from the tip to the frog (handle of the bow) by flexing the elbow (FE) joint, whereas the opposite is called downstroke. From experience, it can be observed that there is a tendency to raise the right shoulder during the upstroke, especially in violin beginners [11].

In comparison to other instrument groups, string players, especially violinists and violists, often complain about shoulder pain $[4,12]$. One possible origin of musculoskeletal disorders of the shoulder girdle could be the elevation of the shoulder while playing the violin. A lot of information about the behavior of the upper limbs concerning an elevated left shoulder as well as abduction/adduction and flexion/extension of the right shoulder is available, e.g. [13,14]. There is almost no literature regarding the elevated right shoulder while bowing, whereby presumably serious damage can also result from frequent and often rapid movements. The impingement syndrome is common among musicians $[5,15,16]$, which may result from increased forces in the glenohumeral $(\mathrm{GH})$ joint caused by playing with a raised shoulder.

For this reason, this study investigates the forces acting on the right $\mathrm{GH}$ joint when playing the violin with the elevated right shoulder and playing with correct posture declared by Medoff in Movement education [9]. It is hypothesized that the resulting forces in the right glenohumeral joint are higher in playing the violin with an elevated right shoulder than in playing in a normal position. This investigation is performed using musculoskeletal simulation via inverse dynamics.

\section{Materials and methods}

In the following study, only the 'legato'-technique playing, i.e., playing with the bow on the strings (as opposed to pizzicato: plucking the strings), is analyzed. To be able to use the expression 'high shoulder', the right shoulder of each subject must be elevated at least $5^{\circ}$ more than the average elevation angle in the sternoclavicular (SC) joint of all trials of each subject in a normal position.

For this study, motion capture data of subjects playing the violin was recorded (Vicon) and then further processed with a musculoskeletal simulation software (AnyBody Modeling System ${ }^{T M}$ ) to calculate the required joint angles and reaction forces.

\subsection{Subjects}

Four subjects (two females and two males), ranging from 22 to 59 years of age (mean 44 years) participated in this study. The test subjects were informed in advance about the measurement procedure, 


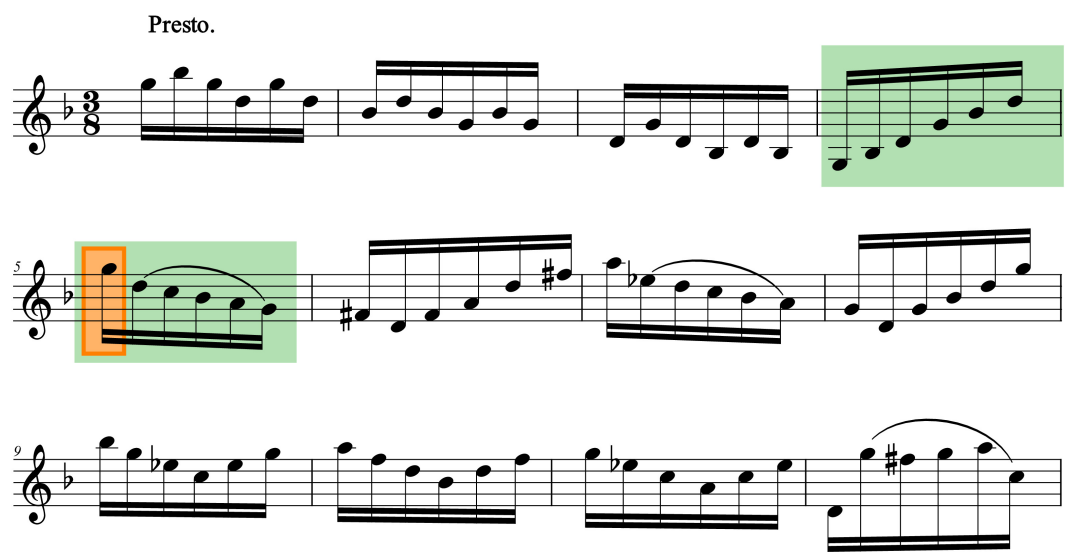

Fig. 1. Excerpt of Bach's G minor sonata (BWV 1001), $4^{\text {th }}$ movement, with the green section considered in this study and the orange marked key g" as highest key. Source: [14], edited by the author.

and a corresponding written consent was obtained concerning voluntary participation. All subjects have experience in playing the violin for at least 16 years. They reported no current right shoulder disorder, but one subject had a lesion in the right shoulder in the past.

Each subject played the first 12 bars from the 4th movement (Presto) of Bach's G minor solo sonata (BWV 1001) [17] three times in normal posture and three times with elevated shoulder. To ensure that the subjects adopted the correct position, they were instructed before each trial to raise their shoulder while playing or playing in the normal position. However, only the green marked passage in Fig. 1 was analyzed in the calculation, since the most considerable string changes occur in these two bars, which can be assumed to be an indication of increased shoulder force.

\subsection{Motion capture data}

For the motion capture recording, the infrared-camera system Vicon (Vicon Motion Systems Ltd, Oxford, UK) within a setup including 12 Vero cameras with a sampling frequency of $250 \mathrm{~Hz}$ was used. To record the kinematic data, the subjects were equipped with 29 markers, which were recorded by the cameras distributed around the room. The standard marker setup according to the AnyBody example model Plug-in-gait Simple from the AnyBody Managed Model Repository was used, but only the markers cranial of the pelvis were considered. Additionally, one marker was attached to the olecranon of each side. The movement of the fingers was omitted due to irrelevance for this study, consequently, no markers were placed there. Also, no markers were placed on the violin or the bow itself. Since the kinematics of the right arm is of major importance, eight markers were fixed from the shoulder to the wrist.

In this paper, the sequences are named with an index for the subject (s) and an index for the trial (t), for example, s2t5. The trials with an odd index ( $\mathrm{t} 1, \mathrm{t} 3$ and $\mathrm{t} 5)$ represent a sequence played in normal position, and sequences with the raised shoulder are labelled with even indices (t2, $t 4$ and $t 6)$.

\subsection{Simulation with AnyBody}

Using the simulation software AnyBody Modeling System ${ }^{\mathrm{TM}}$ (version 7.3.1; AnyBody Technology A/S, 2020), a human model, which reconstructs the motion capture data, was created with the input of the anthropometrics of the subjects and the motion capture data. For this application, the example model Plug-in-gait Simple provided by AnyBody Managed Model Repository was modified (see Fig. 2). 


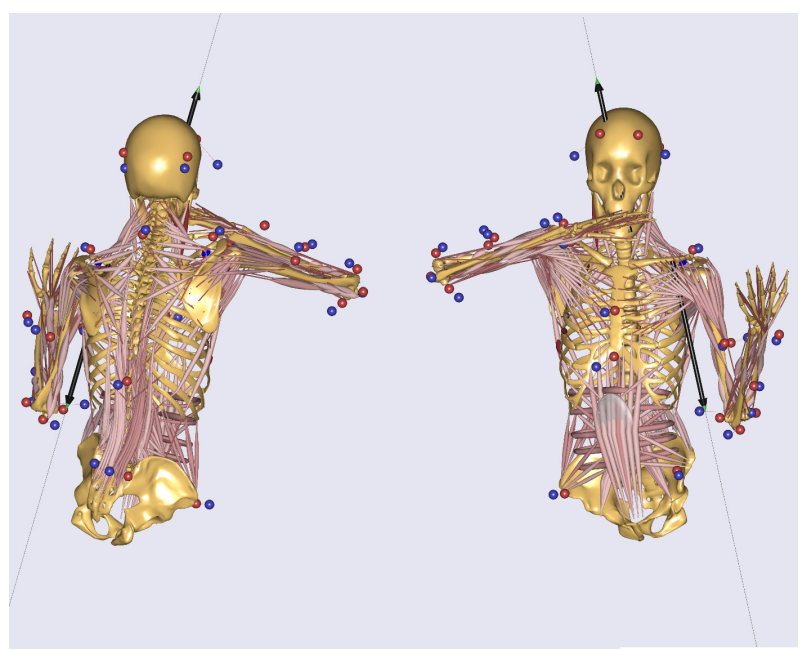

Fig. 2. Modified AnyBody model with the black arrows as a visualization of the weight of the violin and the holding force acting on the chin and the left clavicle. Due to the rotation of the upper body while playing the violin, the black arrows are tilted in the pictures.

Each subject of the study played with its own instrument, following small differences in the masses of the violins. In relation to the bodyweight of the subjects, it was assumed that these differences can be ignored, and thus an estimated average value of $0.6 \mathrm{~kg}$ is assumed for the weight of the violin including the attachments. Two external forces were added to the model to consider the additional weight in the model due to the violin. The force vector acting on an approximated point of the chin illustrates the holding force of the violin with the assumed value of $20 \mathrm{~N}$ [18] opposite to gravity. This force is counteracted by the weight of the violin of about $0.6 \mathrm{~kg}$, including the attachments, and the reaction to the holding force. The average mass of the bow of about $65 \mathrm{~g}$ is negligible compared to the mass of the subjects. Besides, the bow rests on the strings in legato playing, which means that not the entire weight rests on the right arm. Therefore, the weight of the bow is omitted.

To provide a more realistic representation of shoulder forces, Hill-type muscles were used as muscle models in the simulation. Based on [19], not only the active muscle components but also passive components are thus considered. Furthermore, a predefined shoulder rhythm (_RHYTHM_SOFT_) for the arms was used, which contains a movement of the scapula and clavicle depending on the motion of the humerus [20]. In this setting the kinematic rhythms are handled as soft constraints.

Muscle fatigue is not considered in this study because only a short passage is played. Furthermore, since the subjects play the violin for several hours a day, they are used to the movement sequences. Therefore, the maximum synergy approach (MR_MinMaxStrict) in AnyBody was used for muscle recruitment. In this approach, external loads are distributed among several muscles, which results in the fact that not only a single muscle is loaded. Thus, muscle fatigue is not considered. However, the chosen approach does not correspond to physiological conditions since the response time of the muscles is not taken into account. Though this is irrelevant in this study [21].

\subsection{Data processing}

Because a metronome did not guide the subjects during the recording, different playing speeds were detected. To compare the trials to each other, the time axis of each trial was scaled and normalized in all following graphs. 
Elevation angle in right SC joint
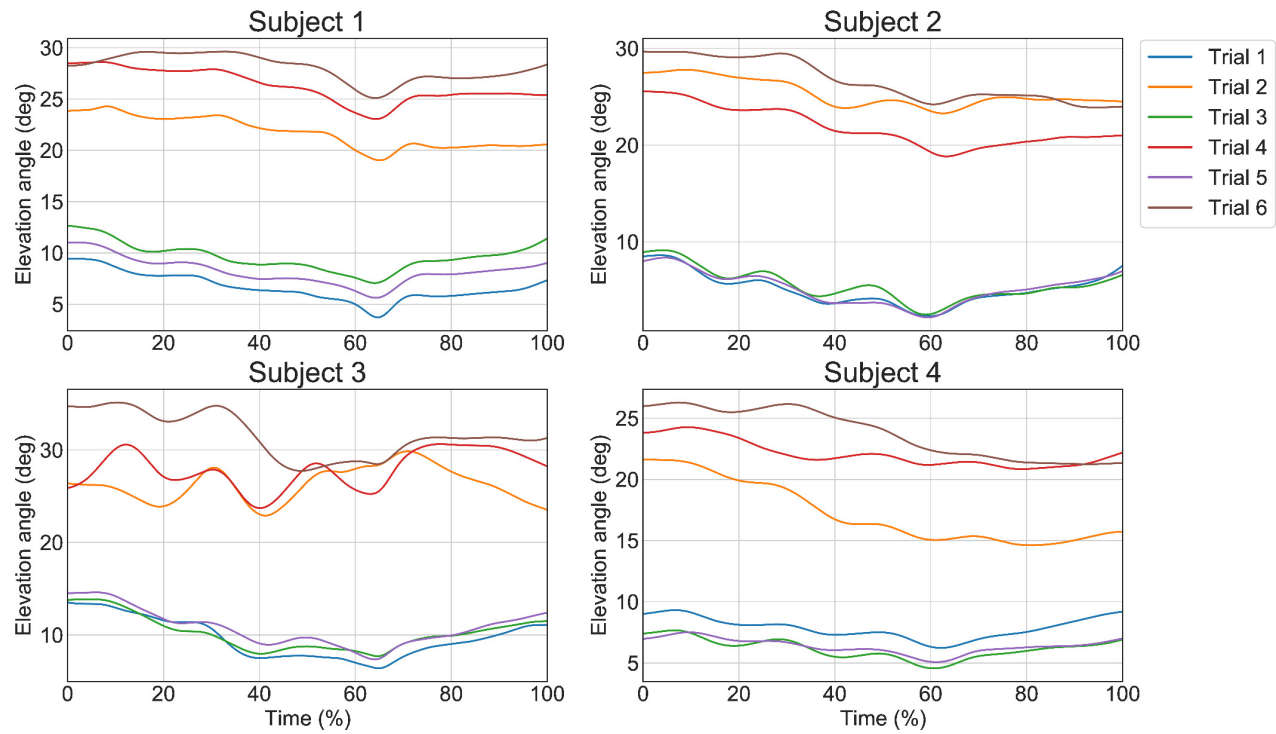

Fig. 3. All trials of each subject regarding the elevation angle in the SC joint.

Based on the flexion angle of the FE joint, the pattern of the keys can be identified, since the upstroke and downstroke can be recognized independently of the position and abduction of the shoulder. An algorithm was applied to the time-dependent data of the angle in the FE joint, which detects peaks in this curve. Based on the first and last relevant peak of the sequence, the time axis was scaled and normalized to a uniform length.

\subsection{Outcome variables}

In the following, the elevation angle Îs describes the deflection of the clavicle in the SC joint.

The force in the GH joint was computed using the software AnyBody. The force components in mediolateral, inferosuperior and anteroposterior directions were also considered, which constitute the total force by vectorial addition.

To outline the differences of the resulting forces between the shoulder positions 'UP' and 'DOWN', the difference between the two medians belonging to the data of one subject was calculated. This value was then set in relation to the absolute value of the median of the relating 'DOWN' data of each subject.

\section{Results}

\subsection{Elevation angles in the right sternoclavicular joint}

To determine the cranial displacement of the right $\mathrm{GH}$ joint and, therefore the state, if the shoulder is raised or not, the angle of the clavicle in the sternoclavicular (SC) joint is considered. Figure 3 shows all curves of each subject playing in the normal position and with elevated shoulder. A distinct difference between the sequences in normal position and shoulder raised is visible.

It is noticeable that the graph of $\mathrm{s} 4 \mathrm{t} 2$ is below the other curves with raised shoulder regarding the values 
Table 1

Force increase of median resulting force in $\mathrm{GH}$ joint from shoulder position low to a high shoulder

\begin{tabular}{cccc}
\hline \multirow{2}{*}{ Subject } & \multicolumn{2}{c}{$\begin{array}{c}\text { Median force (N) } \\
\text { of all trials of }\end{array}$} & $\begin{array}{c}\text { Force increase (\%) from } \\
\text { 'DOWN' to 'UP' }\end{array}$ \\
\cline { 2 - 3 } 'UP' & 'DOWN' & \\
\hline s1 & 381.34 & 259.67 & 147 \\
s2 & 883.50 & 494.64 & 179 \\
s3 & 959.82 & 318.64 & 301 \\
s4 & 779.76 & 330.61 & 236 \\
\hline
\end{tabular}

Resulting force in right $\mathrm{GH}$ joint
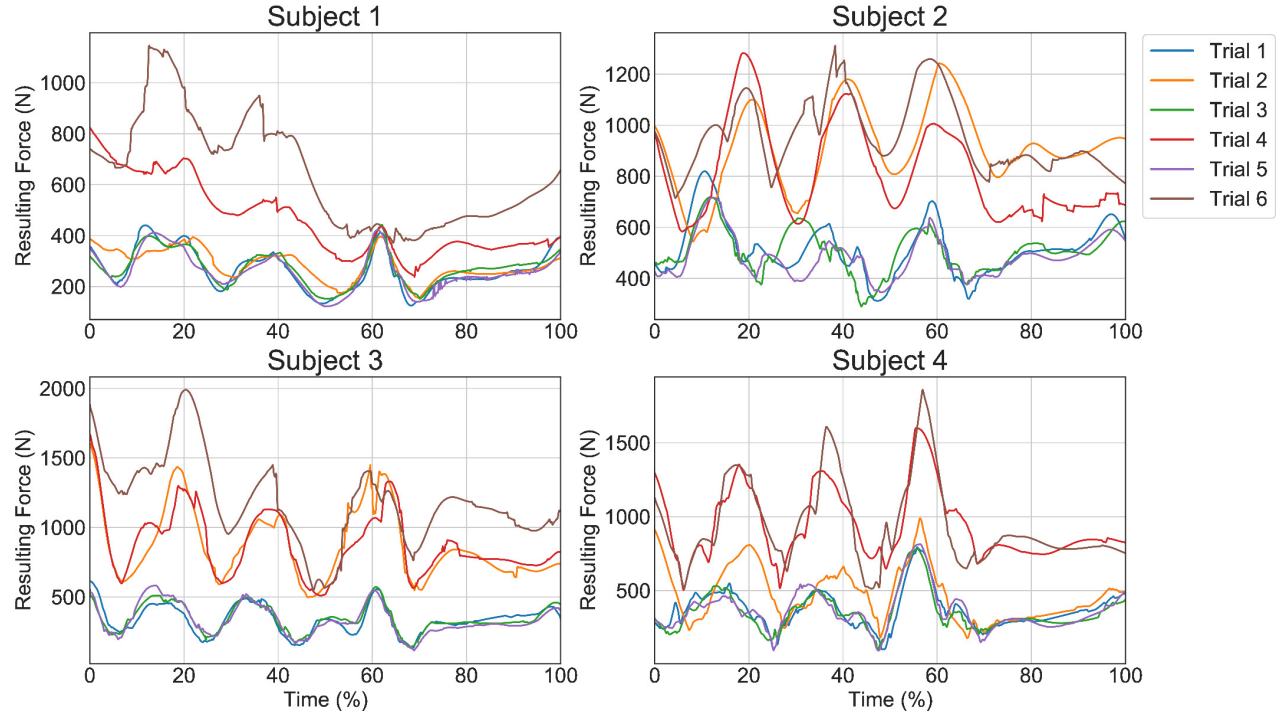

Fig. 4. All trials of each subject regarding the resulting force in the glenohumeral joint.

of the elevation angle. Concerning the trials of s4 the minimum of $\mathrm{s} 4 \mathrm{t} 2$ is $\alpha=14.62^{\circ}$, whereas the mean angle of the three curves in normal position amounts $\alpha=7.16^{\circ}$. Consequently, the minimum angle of $\mathrm{s} 4 \mathrm{t} 2$ is more than the defined difference of $5^{\circ}$ of the average angle in normal position, and thus it can be considered as a trial with an elevated shoulder.

\subsection{Force in the right glenohumeral joint}

All curves of the resulting force in the GH joint for both positions with elevated shoulder and normal position are presented in Fig. 4. The graphs are normalized over time and separated by subjects. Overall, the difference between the trials with the shoulder elevated and in a normal position is visible. The force values are higher at playing with an elevated shoulder than in a normal position. No statement can be made regarding the relationship between the string played and the force in the $\mathrm{GH}$ joint. Concerning the force of the trials of s1 with an elevated right shoulder, it is remarkable, that these forces have a low value. As well as these trials of s1, s4t2 also shows low force values compared to the other 'UP' trials.

Differentiated into the trials with elevated shoulder and the normal position of each subject, the resulting 


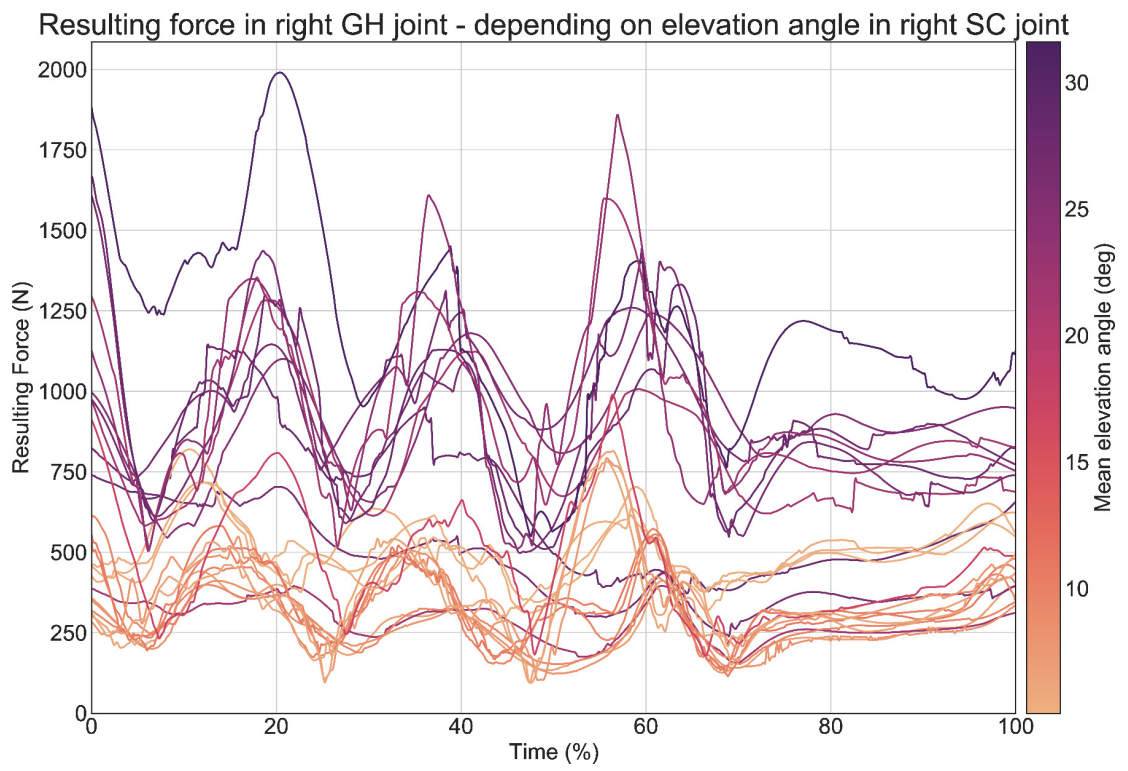

Fig. 5. Resulting Force of all trials with colored dependence of the elevation angle in the SC joint. Light orange represents a low elevation angle whereas dark purple depicts a high elevation angle.

median forces of the 'UP'-trials can be compared directly to the relating 'DOWN'-trial. The values in Table 1 represent a percentage increase in force from the 'DOWN' value to the 'UP' value.

Figure 5 depicts the resulting force of the trials over the sequence. A curve in orange color indicates a low mean elevation angle in the right SC joint, whereas a high angle is marked by a purple curve. Therefore, the relation between the elevation angle of the right shoulder and the resulting force in the GH joint during the sequence is demonstrated. It is noticeable that the orange lines and thus the curves of a low mean elevation angle are placed at the bottom of the graph, while the most purple curves of the low force are located significantly above the orange ones in most cases. In this kind of illustration, the curves with low forces mentioned before during playing with the high shoulder can be identified as purple lines in the region of the orange-colored lower force lines.

By distributing the resulting force into the three principal directions (mediolateral, inferosuperior, and anteroposterior) and the two data sets 'UP' and 'DOWN' for each subject, it is evident that in all subjects the absolute median force value for a raised shoulder is higher than in a normal position.

\section{Discussion}

This study investigates whether a higher force results in the right GH joint when the right shoulder is elevated during playing the violin. The results indicate that this force is higher when playing with a raised shoulder than in normal posture.

A detailed view on Fig. 3 reveals a kink in most of the curves at approximately $65 \%$ of the sequence. In the example of s1t4, this angle is set in relation to the corresponding abduction angle in the GH joint and the flexion angle in the FE joint. Due to the small kink in the SC curve, this line is displayed on a second axis. It is evident that this kink coincides with the maximum of the flexion angle in the FE joint and the minimum of the GH joint. At this location in the sheet music, the key g" (see Fig. 1, orange marked key) is played within a downstroke to play the following five keys within a single bow length at upstroke. Thus, 


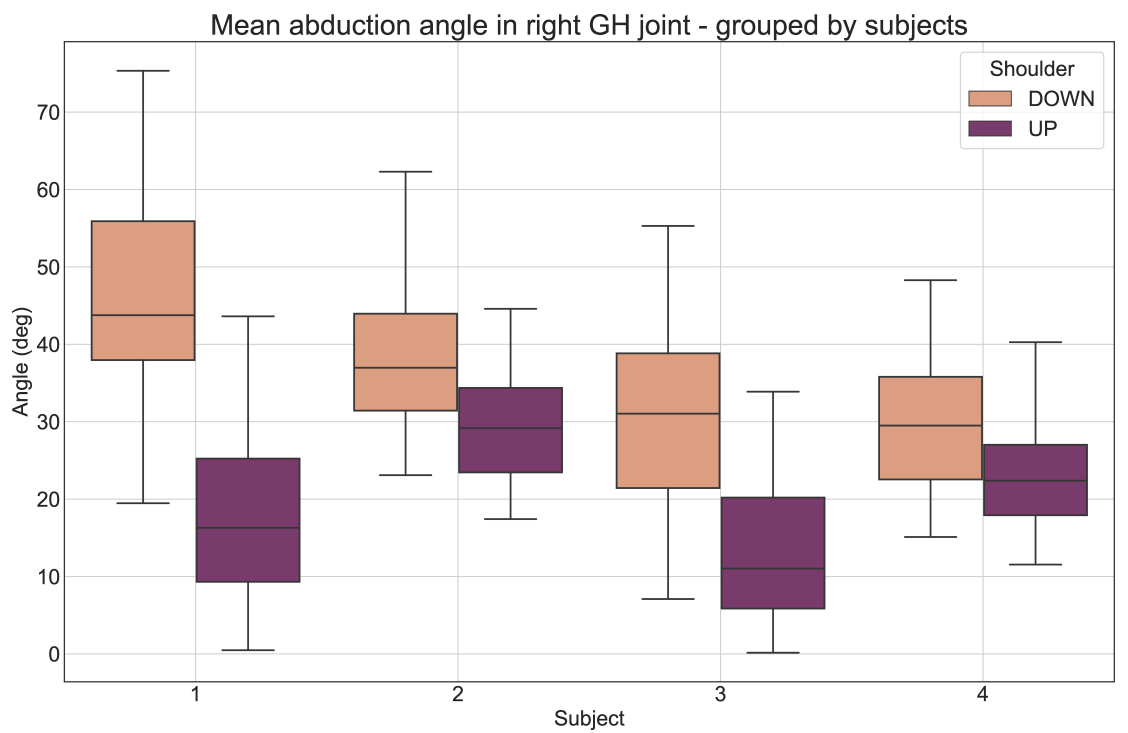

Fig. 6. Boxplot of the abduction angles in the GH joint differentiated in the shoulder positions 'UP' and 'DOWN' of each subject.

this downstroke is followed by a lowering of the right elevated shoulder. In the subsequent upstroke, the shoulder is usually raised back to the typical deflection angle.

In most of the sequences, wavelike patterns can be identified in the elevation angle in the SC joint, as clearly shown in the example of s3t4 (see Fig. 3). This is more intense in some trials than in others. The minima of the angle of the FE joint correspond approximately to the maxima of the angle of the SC joint regarding the time. Thus, the correlation of the shoulder elevation with flexion of the FE joint can be determined. This flexion is followed by the extension and, therefore, the depression of the shoulder.

Also, it is conspicuous that the curves in the force plots have step changes (see Fig. 4). This phenomenon can be explained through the muscle recruitment of the AnyBody model. When the sign of the moment arm changes from positive to negative, the relevant muscles abruptly switch off, causing the steps in the force curve [21]. Furthermore, the AnyBody model does not consider muscle dynamics, whereby steps in the force curves also can occur.

Respecting the data of the resulting force in the GH joint, it is noticeable that the graph of $\mathrm{s} 4 \mathrm{t} 2 \mathrm{has}$ low force values at some points compared to the other graphs of subject $s 4$ with the shoulder raised (see Fig. 4). This can be explained by the angle in the SC joint during this trial (see Fig. 3). Because the subject did not raise the shoulder very high at the beginning of the considered section and lowered it more and more during the sequence, a low force occurs in the GH joint.

There is a relation between the glenohumeral abduction angle and the resulting force in the GH joint. With an increase of the abduction angle, the force also increases [22,23]. In Fig. 6, the abduction angle of the GH joint is displayed in the form of a boxplot. The values are divided into normal position and elevated shoulder and grouped by each subject. Remarkably, the abduction angle when playing with a raised shoulder is lower for all subjects than in a normal position. According to [22,23], the result is that the force in the GH joint with a lower abduction angle should have a lower value. However, the force in the shoulder joint is significantly higher with a raised shoulder than in playing in a normal position for all subjects, although the abduction angle in the GH joint has a lower value with a raised shoulder. Considering the small difference of $s 1$ between the resulting force from shoulder raised to normal position, it is concluded that the abduction angle in the GH joint still results in a force reduction. 
Regarding the force values in the right $\mathrm{GH}$ joint, there is an increase in force from playing the violin in a normal position to playing with a raised shoulder in most cases (see Table 1 and Fig. 4). The deviating curves are explained by considering the $\mathrm{SC}$ joint and $\mathrm{GH}$ joint angle during violin playing with an elevated shoulder (see Fig. 6).

In contrast to most literature sources existing in the sector of Performing Arts Medicine, this study applies calculations, whereas many other literature sources mainly provide statistics by surveys. As the results in this paper are not measured directly in vivo but generated using musculoskeletal simulation, there may be deviations from real values. Furthermore, many parameters may contribute to the result, but they are not investigated in detail here. This includes, inter alia, the abduction angle in the GH joint, or the stroke length for a single key.

Because the calculations in this study are based on a musculoskeletal system and the muscle forces were not measured directly, there may be differences compared to the real force values. As the model applied in AnyBody does not exactly correspond to the human body, this may contribute to these differences. Furthermore, inaccuracies of the results may be caused by the measurement system, the data processing, the chosen settings in AnyBody and the fact that the subjects were not guided by a metronome. The number of only four subjects may also be a limitation due to time constraints, but the results indicate a noticeable trend towards higher forces when playing the violin with a raised shoulder.

Overuse syndrome in the shoulder girdle is very frequent, especially among string players [3], resulting from repetitive movements. There is no standard definition, but overuse syndrome is often associated with pain and loss of function in muscle groups and ligaments due to excessive use [24]. Repeated movements with a high shoulder while playing the violin lead to the assumption that the effects of the overuse syndrome can be more severe than playing in a normal position.

\section{Conclusion}

In this study, the relationship between an elevated right shoulder and the resulting joint forces in the right GH joint in playing the violin was investigated. The hypothesis that the resulting force in the right GH joint is higher in playing the violin with a raised right shoulder compared to playing in a normal position was confirmed by the noticeable trend of the results.

There is already a lot of literature regarding statistics in form of surveys, but further research is still required to investigate the relating biomechanical data. Furthermore, future research will conduct the relationship of muscle forces as well as the lengths of the involved muscles with the movement sequences.

Based on the findings of this study, it is important to pay attention if the right shoulder is elevated while playing the violin.

\section{Acknowledgments}

The authors thank the participants in this study. Further thanks go to Evelyn Husser, who supported the implementation of the study with her ideas and assistance.

\section{Conflict of interest}

None to report. 


\section{References}

[1] Lee HS, Park HY, Yoon JO, Kim JS, Chun JM, Aminata IW, et al. Musicians' medicine: Musculoskeletal problems in string players. Clinics in Orthopedic Surgery. 2013 Aug; 5(3): 155-160. doi: 10.4055/cios.2013.5.3.155.

[2] Rensing N, Schemmann H, Zalpour C. Musculoskeletal demands in violin and viola playing: A literature review. Medical Problems of Performing Artists. 2018 Dec; 33(4): 265-274. doi: 10.21091/mppa.2018.4040.

[3] Fry HJ. Incidence of overuse syndrome in the symphony orchestra. Medical Problems of Performing Artists. 1986; 1(2): 51-55.

[4] Zetterberg C, Backlund H, Karlsson J, Werner H, Olsson L. Musculoskeletal problems among male and female music students. Medical Problems of Performing Artists. 1998; 13(4): 160-166.

[5] Rickert D, Barrett M, Halaki M, Driscoll T, Ackermann B. A study of right shoulder injury in collegiate and professional orchestral cellists: An investigation using questionnaires and physical assessment. Medical Problems of Performing Artists. 2012 Jun; 27(2): 65-73. doi: 10.21091/mppa.2012.2014.

[6] Fry HJ, Rowley GL. Music related upper limb pain in schoolchildren. Annals of the Rheumatic Diseases. 1989 Dec; 48(12): 998-1102. doi: 10.1136/ard.48.12.998.

[7] Shoup D. Survey of performance-related problems among high school and junior high school musicians. Medical Problems of Performing Artists. 1995; 10: 100-105.

[8] Kaufman-Cohen Y, Ratzon NZ. Correlation between risk factors and musculoskeletal disorders among classical musicians. Occupational Medicine. 2011 Jan; 61: 90-95. doi: 10.1093/occmed/kqq196.

[9] Medoff LE. The importance of movement education in the training of young violinists. Medical Problems of Performing Artists. 1999; 14(4): 210-219.

[10] Schünke M, Schulte E, Schumacher U, Voll M, Wesker K. Prometheus: Allgemeine Anatomie und Bewegungssystem. Stuttgart: Georg Thieme Verlag, 2005.

[11] Volta E, Mancini M, Varni G Volpe G. Automatically measuring biomechanical skills of violin performance: an exploratory study. Proceedings of the 5th International Conference on Movement and Computing, MOCO 2018, 2018 Jun, Genoa Italy. New York: Association for Computing Machinery; 2018. Article 16.

[12] McCrary JM, Halaki M Ackermann BJ. Effects of physical symptoms on muscle activity levels in skilled violinists. Medical Problems of Performing Artists. 2016 Sep; 31(3): 125-131(7). doi: 10.21091/mppa.2016.3024.

[13] Rabuffetti M, Converti RM, Boccardi S, Ferrarin M. Tuning of the violin-performer interface: An experimental study about the effects of shoulder rest variations on playing kinematics. Medical Problems of Performing Artists. 2007 Jun; 22(2): 58-66. doi: 10.21091/mppa.2007.2013.

[14] Steinmetz A, Seidel W, Niemier K. Shoulder pain and holding position of the violin: A case report. Medical Problems of Performing Artists. 2008 Jun; 23(2): 79-81(3). doi: 10.21091/mppa.2008.2015.

[15] Kulig K, Mulroy SJ. Shoulder joint kinetics during the push phase of wheelchair propulsion. Clinical Orthopaedics and Related Research. 1998; 354: 132-143. doi: 10.1097/00003086-199809000-00016.

[16] Pyne SW. Diagnosis and current treatment options of shoulder impingement. Current Sports Medicine Reports. 2004; 3(5): 251-255. doi: 10.1007/s11932-004-0049-2.

[17] Bach JS. Violin Sonata No.1 in G minor (BWV 1001). In: 6 Violin Sonatas and Partitas. 1720.

[18] Obata S, Kinoshita H. Chin force in violin playing. European Journal of Applied Physiology. 2012 Jun; 112: 2085-2095. doi: 10.1007/s00421-011-2178-7.

[19] Aurbach M, Spicka J, Süß F, Dendorfer S. Evaluation of musculoskeletal modelling parameters of the shoulder complex during humeral abduction above $90^{\circ}$. Journal of Biomechanics. 2020 Jun; 106: 109817. doi: 10.1016/j.jbiomech.2020. 109817.

[20] Anybody Technology. Shoulder Rhythm. Available: https://www.anybodytech.com/download.html?did=publications.files \&fname=ShoulderRhythmReport.pdf.

[21] AnyBody Technology. Lesson 5: Min/Max Muscle Recruitment. Available: https://anyscriptorg/tutorials/MuscleRecruit ment/lesson5.html. [Accessed: 22.03.2021].

[22] Konrad GG, Jolly JT, Labriola JE, McMahon PJ, Debski RE. Thoracohumeral muscle activity alters glenohumeral joint biomechanics during active abduction. Journal of Orthopaedic Research. 2006 Mar; 24(4): 748-756. doi: 10.1002/jor. 20062 .

[23] Nikooyan AA, Veeger HEJ, Westerhoff P, Graichen F Bergmann G, van der Helm FCT. Validation of the Delft Shoulder and Elbow Model using in-vivo glenohumeral joint contact forces. Journal of Biomechanics. 2010 Nov; 43(15): 3007-3014. doi: 10.1016/j.jbiomech.2010.06.015.

[24] Fry HJ. The treatment of overuse syndrome in musicians. Results in 175 patients. Journal of the Royal Society of Medicine. 1988; 81(10): 572-575. doi: 10.1177/014107688808101007. 